Research Article

\title{
A High-Power Pulse Generator Based on Pulse Forming Network and Linear Transformer
}

\author{
Mingjia Li $\mathbb{D}^{1},{ }^{1}$ Qiang Kang, ${ }^{2}$ Jie Tan, ${ }^{2}$ Min Luo, ${ }^{2}$ and Fei Xiang ${ }^{2}$ \\ ${ }^{1}$ Institute of Nuclear Physics and Chemistry, China Academy of Engineering Physics, Mianyang 621900, China \\ ${ }^{2}$ Science and Technology on High Power Microwave Laboratory, Institute of Applied Electronics, \\ China Academy of Engineering Physics, Mianyang 621900, China \\ Correspondence should be addressed to Mingjia Li; caeplmj@126.com
}

Received 9 June 2020; Accepted 6 January 2021; Published 28 January 2021

Academic Editor: Dieter H.H. Hoffmann

Copyright (c) 2021 Mingjia Li et al. This is an open access article distributed under the Creative Commons Attribution License, which permits unrestricted use, distribution, and reproduction in any medium, provided the original work is properly cited.

With the development of high-power microwave technology, the output power of the pulse generator is required more and more higher. In this paper, it is realized by increasing the output power of the module while the output impedance of the module changes little. The module of the generator is based on pulse forming network (PFN) and linear transformer (LT). Four Blumlein PFNs with arc-type configuration and $24 \Omega$ characteristic impedance were connected symmetrically to the primary coil of the LTD and driven by two identical laser triggered spark switches to ensure four Blumlein PFNs synchronizing operation. On this basis, a two-stage high-power pulse generator based on PFN-LT is developed. The following technical parameters of the generator were achieved on a $12 \Omega$ high-power solid resistor: output voltage amplitude of $\sim 250 \mathrm{kV}$ and output power of $\sim 5.2 \mathrm{GW}$ at a repetition rate of $5 \mathrm{~Hz}$

\section{Introduction}

A repetitive long-pulse power generator [1] based on PFNLT has been developed by China Academy of Engineering Physics (CAEP) in China. The generator has the characteristics of compact structure, modularization, and repetitive operation. The quasirectangular pulse with a pulse width of several hundred nanoseconds was formed by one, but only one energy compression can directly drive the load.

With the development of high-power microwave, the output power of the generator is required more and more higher [2-6]. As for the generator based on PFN-LT, there are two methods to achieve it. The first way can be realized by increasing the number of stages [7]. However, with the increase in the number of stages, the output impedance of the device increases, which does not meet the requirement of the related load. The other way is to increase the output power of the module, while the output impedance of the module changes little.
In this paper, a module, based on PFN and LT, is designed. The output power of the module is about twice as much as that of the module described in [1]. On this basis, a two-stage high-power pulse generator with two modules is developed.

\section{Design of High-Power Pulse Module}

2.1. General Review. Each high-power pulse module is composed of four Blumlein pulse forming networks (PFNs), two spark switches, and a linear transformer (LT). Configuration of the long-pulse power module is shown in Figure 1.

Four Blumlein PFNs, whose characteristic impedance is all $24 \Omega$, are connected symmetrically with the LT's primary coil and driven by two spark switches to provide synchronic operation. In order to compact structure, less connected inductance, and better output pulse waveform, the structure of the Blumlein PFN is designed to be arc-type 


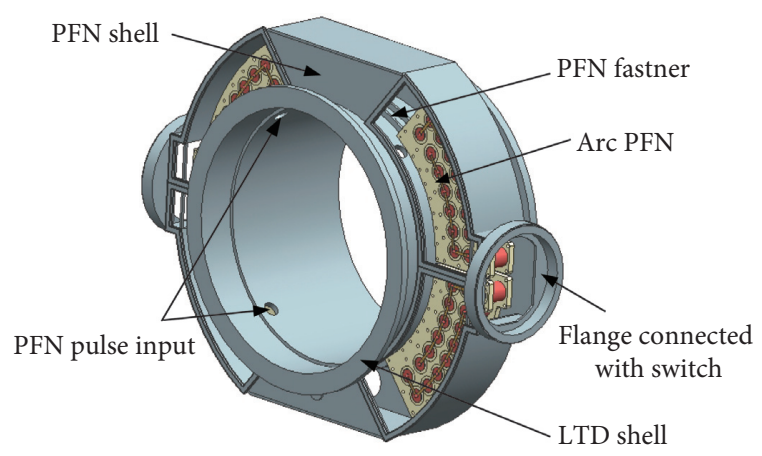

(a)

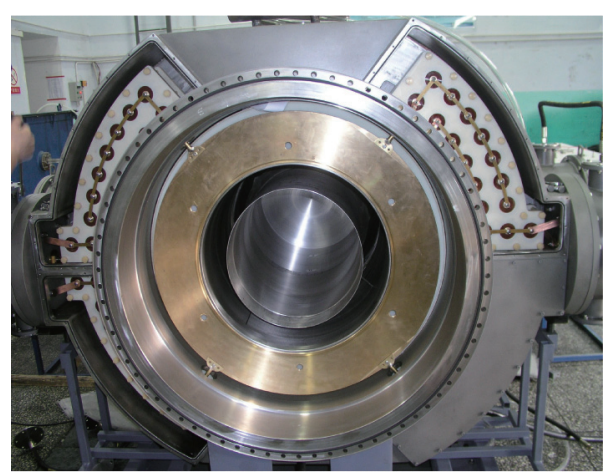

(b)

Figure 1: Configuration of the long-pulse power module: (a) 3D model; (b) photo.

configuration, as shown in Figure 2. The insulation boards are made of nylon, which has corresponding holes for supporting the capacitors. The arc-type PFNs are fixed on the LT shell by bolts as a whole. Four arc-type PFNs are symmetrically placed along the vertical section of the LT's axis, and a spark switch (laser triggered gas switch, detailed as follows) is laid between every two arc-type Blumlein PFNs. The high-voltage pulses formed by PFNs are symmetrically fed into the LT through the four equidistant tabs located at $\pm 45^{\circ}$ and $\pm 135^{\circ}$ of the LT connecting plate rim, respectively (shown in Figure 1(b)). With such a structure, the current uniformity of the LT's primary coil is much better than that of the single point feed [8], which is beneficial to establish uniform magnetic field and reduce the waveform distortion in the process of inductive voltage addition.

2.2. Blumlein PFN. A quasirectangular pulse waveform can be achieved by using PFN, and PFNs consisting of identical inductors and capacitors connected in cascades by ladder style are generally used $[9,10]$. As for Blumlein PFN, it has the advantage that the peak output pulse voltage is equal to the charging voltage, which reduces the system insulation requirements. In this paper, Blumlein PFNs are designed to generate a quasirectangular pulse with a voltage of $130 \mathrm{kV}$ and a pulse width of $160 \mathrm{~ns}$. The design parameters of the Blumlein PFN are shown in Table 1. The equivalent schematic of four parallel Blumlein PFNs in each module is shown in Figure 3. While the section capacitor charged with $40 \mathrm{kV}$, the simulated output pulse waveform of the Blumlein PFN is shown in Figure 4(a). The amplitude of the voltage pulse is about $40 \mathrm{kV}$ with a width (FWHM) of $160 \mathrm{~ns}$. It can be seen that the simulated waveform has no obvious reflection, which shows that the impedance of each Blumlein PFN is about $24 \Omega$.

In engineering design, the two single PFNs of the Blumlein PFN are used as different arc-type configurations, as shown in Figure 5.7 section capacitors of one single PFN are closely connected by the arc-type section inductor and distributed on the arc with a radius of $605 \mathrm{~mm}$. The $8^{\text {th }}$ section capacitor and the $7^{\text {th }}$ section capacitor are connected

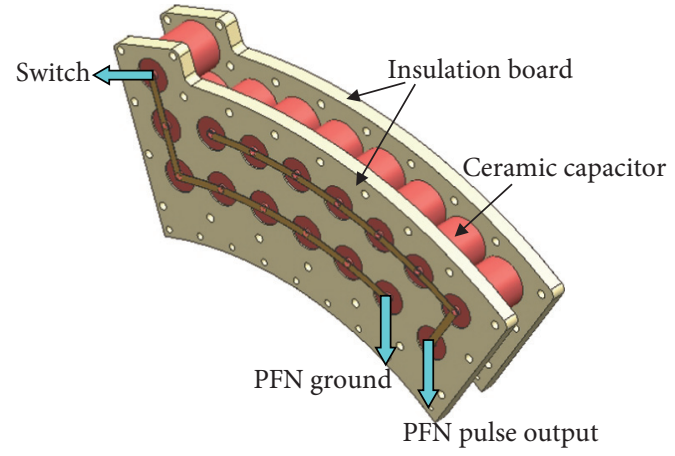

FIgURE 2: Configuration of arc-type Blumlein PFN.

TABLE 1: Design parameters of the Blumlein PFN.

\begin{tabular}{lc}
\hline Design parameters & Values \\
\hline Number of sections $(n)$ & 8 \\
Forming pulse width $(\tau / \mathrm{ns})$ & $\sim 160$ \\
Characteristic impedance $(Z / \Omega)$ & 24 \\
Section capacitance $\left(C_{0} / \mathrm{pF}\right)$ & 866 \\
Section inductance $\left(L_{0} / \mathrm{nH}\right)$ & 100 \\
\hline
\end{tabular}

in a straight line, whose direction is coincided with the arc radius' direction. 6 section capacitors of the other single PFN are closely connected by the arc-type section inductor and distributed on the arc with a radius of $532 \mathrm{~mm}$. The 6th, 7th, and 8th section capacitors are connected in a straight line, whose direction is opposite to the arc radius' direction. The angle in a circular segment of the Blumlein PFN is $50^{\circ}$.

The ceramic capacitor $(2600 \mathrm{pF}, 50 \mathrm{kV}$, size: $\Phi 65 \mathrm{~mm} \times 32 \mathrm{~mm}$ ) with a high relative permittivity is selected as the section capacitor because of its size, voltage capability, and low self-inductance. Three ceramic capacitors are connected in series to form a section capacitor unit. Since the maximum operating voltage of the ceramic capacitors is $50 \mathrm{kV} \mathrm{DC}$, the three series unit is expected to have the withstand voltage more than $150 \mathrm{kV}$ DC. The copper strips with a rectangular cross-sectional shape were chosen as section inductor. The length of one section's copper strip is 


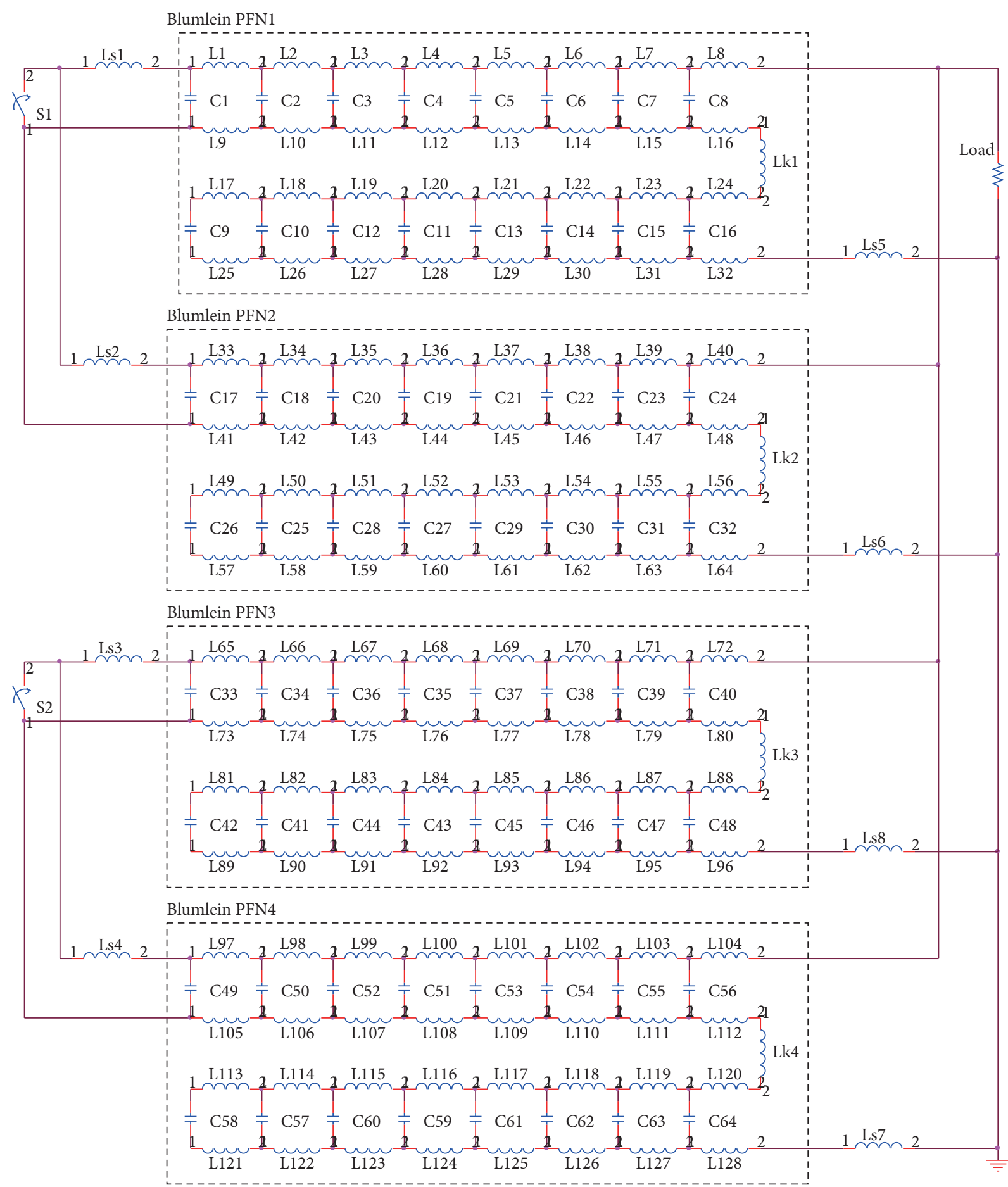

FIgURE 3: Equivalent schematic of four parallel Blumlein PFNs in each module. $L_{1}$ to $L_{128}$ : section inductance, $\sim 60 \mathrm{nH} ; L_{k 1}$ to $L_{k 4}:$ PFN connected inductance; $\mathrm{C}_{1}$ to $\mathrm{C}_{6}$ : section capacitance, $\sim 866 \mathrm{pF} ; L_{s 1}$ to $L_{s 4}$ : switch inductance (includes connected inductance), $\sim 100 \mathrm{nH} ; L_{s 5}$ to $L_{s 8}$ : connected inductance, $\sim 100 \mathrm{nH}$; $\mathrm{S} 1$ and S2: spark switch; load: resistive load, $6 \Omega$.

about $66 \mathrm{~mm}$, which is approximately equal to the diameter of the capacitor, and the size of the copper strip's cross section is $10 \mathrm{~mm} \times 1 \mathrm{~mm}$. The inductance of the section inductor is estimated to be $51.3 \mathrm{nH} \times 2=102.6 \mathrm{nH}$ from the theoretical evaluation [11].

The typical testing output pulse waveform of a single Blumlein PFN with arc-type configuration is shown in
Figure 4(b), while the section capacitor is charged with $40 \mathrm{kV}$ and the resistive load is about $24 \Omega$. One output pulse with a rise time of $55 \mathrm{~ns}$, a width (FWHM) of $180 \mathrm{~ns}$, and a flat time of $100 \mathrm{~ns}$ is obtained. The experimental waveform is not completely consistent with the simulated waveform, such as FWHM and rise time. The main reasons leading to this difference are as follow. First, the calculated value of the 


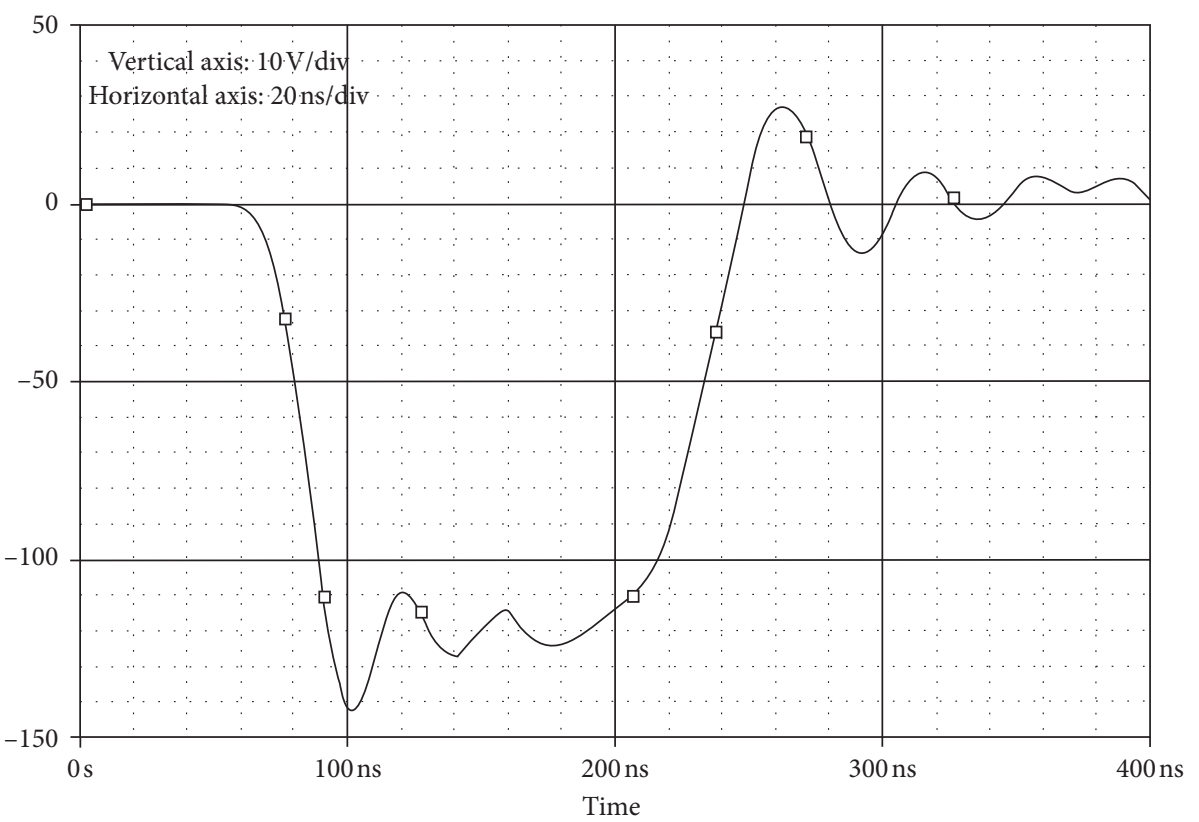

(a)

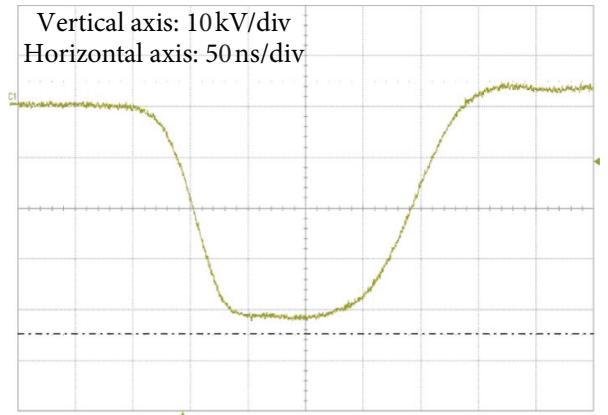

(b)

FIgURE 4: Output waveform of Blumlein PFN: (a) simulated result; (b) typical testing waveform.

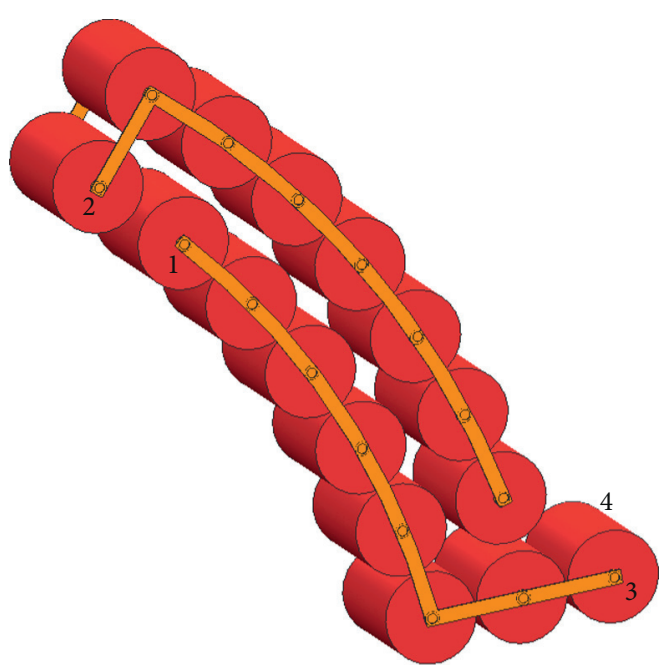

Figure 5: Layout of the Blumlein PFN. 1, connect to LT; 2, connect to ground; 3, connect to anode of the switch; 4, connect to cathode of the switch. 
section inductance is too small by using the formula. In fact, the actual value of the section inductance is about $58 \mathrm{nH} \times 2=116 \mathrm{nH}$, which is measured with a precision digital bridge. Then, the parameters of the two PFNs are not completely consistent, which causes the output pulses of the single PFN different.

2.3. High-Voltage Spark Switch. The high-voltage spark switch in this paper is one kind of laser-triggered gas switch (LTGS), as shown in Figure 6. The switch adopts a coaxial structure, which can reduce the connected inductance and restrain the arc channel on the center of the switch. The two main electrodes are hemispherical. The anode electrode is connected with the point 3 of Blumlein PFNs through an anode rod, and the cathode electrode is connected with the point 4 of Blumlein PFNs through a cathode cylinder. The main body of the electrodes is made of stainless steel (1Cr18Ni9Ti) and has copper tungsten inserts at the center of the anode, where the arc is usually formed. In the middle of the cathode, there is a through-hole, through which a laser beam is injected to trigger the switch. The switch gap distance is adjustable from $10 \mathrm{~mm}$ to $25 \mathrm{~mm}$.

The insulation gas is nitrogen $\left(\mathrm{N}_{2}\right)$. The gas pressure in the switch is in a range of $0-0.4 \mathrm{MPa}$. A gas circulation system with a closed-loop pipeline is used to realize the gas circulation, which can blow high-temperature gas and impurity away from the discharging region and speed up the recovery time of the switch.

2.4. Linear Transformer. Linear transformer is a single turn $1: 1$ pulse transformer in coaxial configuration. The magnetic core of each linear transformer has 15 separate rings, which are wound with $25 \mu \mathrm{m}$ thick Fe-based amorphous film sandwiched in a $1 \mu \mathrm{m}$ thick $\mathrm{SiO}_{2}$ coat insulation. The rings are moulded into a glass fiber compound to stabilize the turns and isolate the rings from each other. $\mathrm{Br}$ of the magnetic core is about $1.2 \mathrm{~T}$, and the corresponding squareness ratio of the hysteresis loop $(\mathrm{Br} / \mathrm{Bs})$ is 0.8 , where $\mathrm{Bs}$ and $\mathrm{Br}$ correspond to the saturation induction and remanent induction of the magnetic core, respectively.

\section{Structure of Pulse Generator}

The pulse generator includes two modules. The structure of the generator is shown in Figure 7. The pulse generator cavity is filled up with transformer oil. In order to reduce the reflection of the pulse waveform and improve the efficiency, the characteristic impedance of each coaxial transmission line, which is composed of the primary coil and the secondary coil, must be matched, and the radius of the secondary coil requires to be decreased as the number of stage increases. The secondary coil of the LT is stepped down at each end of the module. The impedance of the first and second coaxial transmission lines is $6 \Omega$ and $12 \Omega$, respectively.

A frequency quadrupled Nd:YAG laser with a wavelength of $266 \mathrm{~nm}$ is used to trigger all four gas switches. At $266 \mathrm{~nm}$, the laser outputs a $10 \mathrm{~ns}$ pulse with a maximum

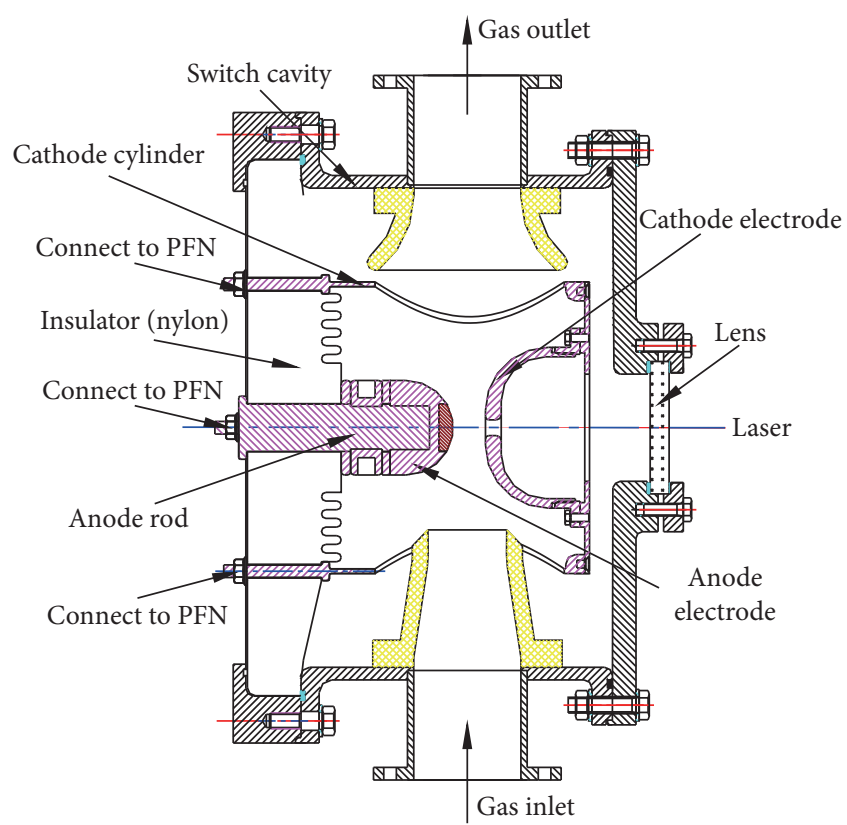

FIgURE 6: Cross-sectional view of the spark switch.

output energy of $30 \mathrm{~mJ}$. When triggering the switch, the laser was fired coaxially into the switch. The optical path to the switch and the switch layout are shown in Figure 8.

The working principle of the pulse generator is described as follows. After the capacitors of the PFNs are charged up to $130 \mathrm{kV}$, four laser beams produced by the laser trigger the four spark switches of the pulse generator synchronous. Then, two homologous pulses are generated and delivered into the primary coils of the linear transformers. After that, the pulses are coupled to a common secondary coil and added. Finally, a high output voltage pulse is obtained on the load.

For the sake of measuring the output voltage of the generator, a measuring section is added between the generator and the load. The measuring section is a coaxial transmission line, whose characteristic impedance is matched to the output impedance of the generator. A capacitive voltage divider made of copper clad laminate is placed on the inner surface of the housing.

\section{Experimental Results}

The global view of the assembled pulse generator is shown in Figure 9. The load is a nominal $12 \Omega$ high-power solid resistor. A capacitive voltage divider plus secondary resistance attenuators was applied to measure the output voltage waveform of the generator.

The typical voltage waveform of the generator output pulse is shown in Figure 10, which comprises 5 pulses at a repetition rate of $5 \mathrm{~Hz}$. The average amplitude of the voltage pulse is about $245 \mathrm{kV}$. Meanwhile, the actual value of the solid resistor is about $11.4 \Omega$, which is measured with a precision digital bridge. The output power is calculated to be about $5.2 \mathrm{GW}$, which is corresponding to the power of four 


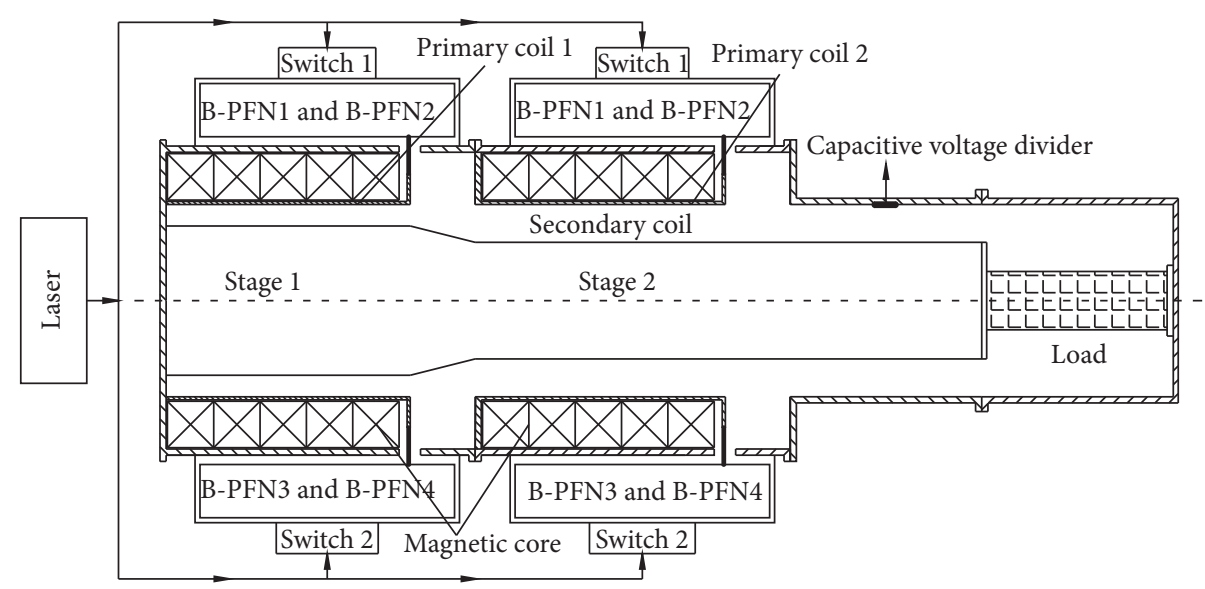

Figure 7: Planform of the pulse generator.

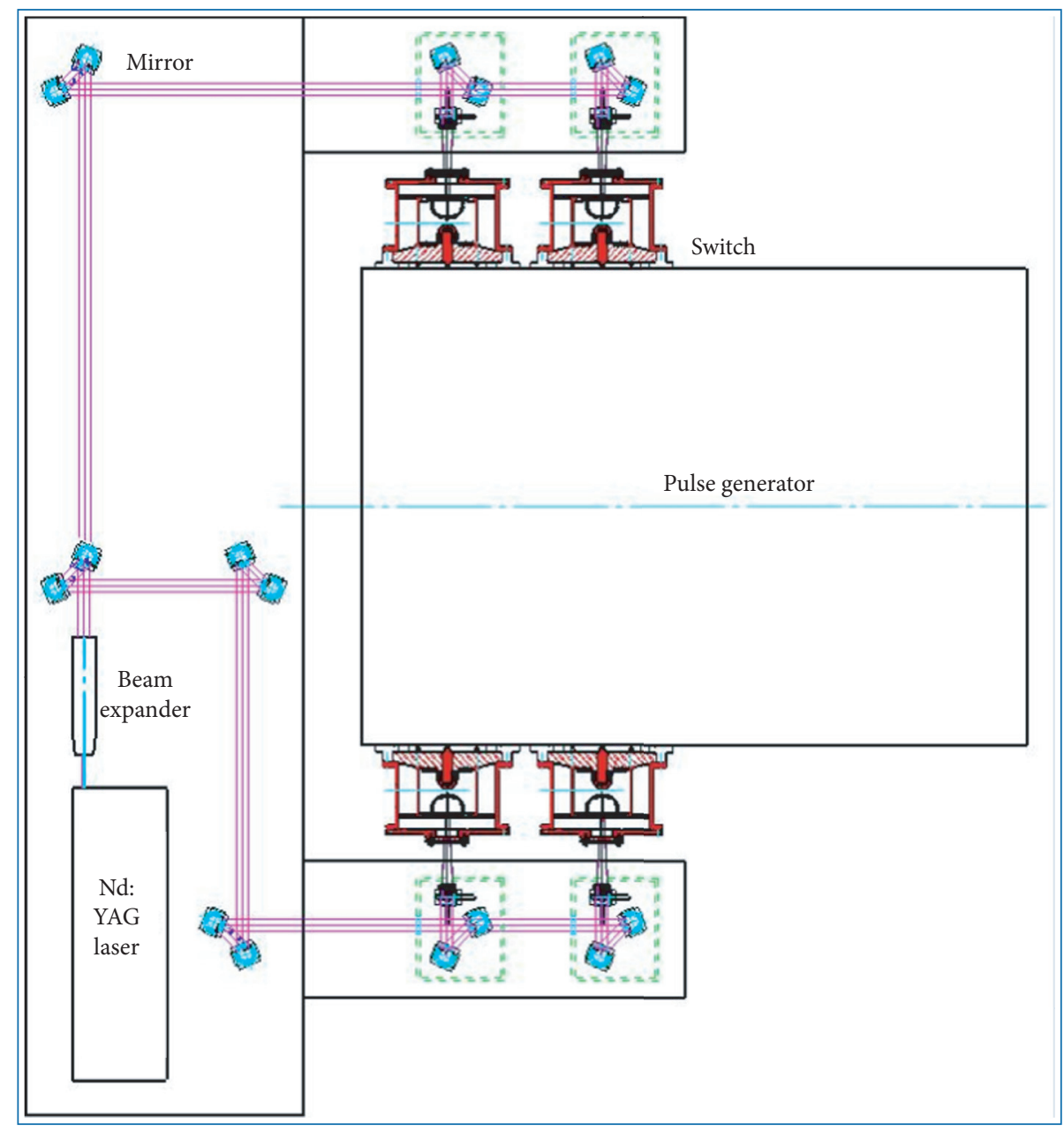

FIgURE 8: Schematic of laser triggers system.

modules of the generator in [1]. The full width (FWHM) of the voltage is tested to be about $180 \mathrm{~ns}$.

The waveform of the generator is not as good as that of single Blumlein PFN. The following are some of the reasons that caused the result. First of all, the output impedance of the generator is about $12 \Omega$, only half of that of a single PFN. With the increase in the number of the modules, the output impedance becomes larger, and the output pulse waveform is expected to be better. Secondly, due to the large volume of high-power solid resistor, the existence of its stray 


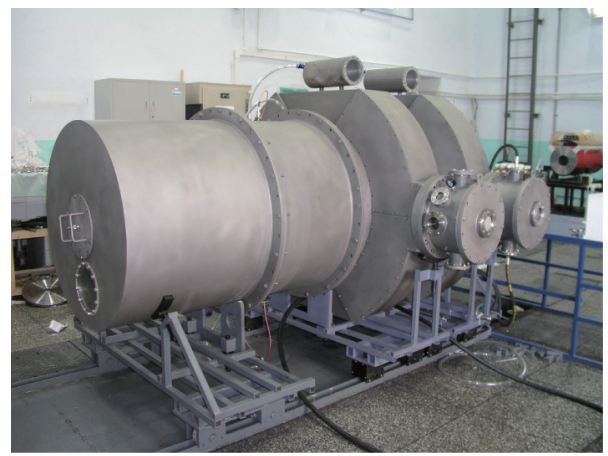

FIGURE 9: Photograph of the pulse generator.

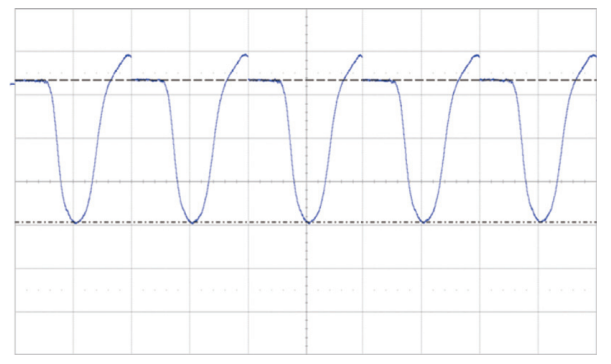

(a)

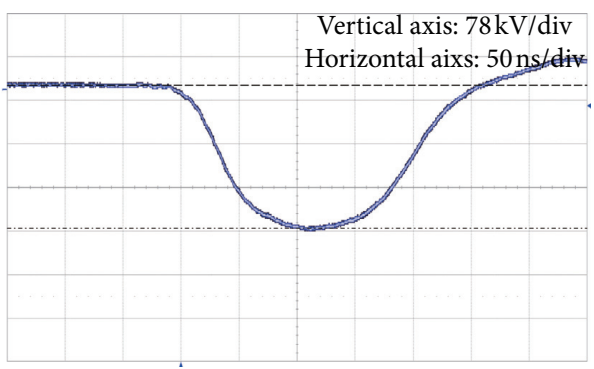

(b)

Figure 10: Typical voltage output waveform of the long-pulse power generator: (a) sequence waveforms; (b) overlay waveforms.

capacitance and stray inductance will also lead to a worse waveform.

\section{Conclusion}

In this paper, a repetitive high-power pulse generator based on PFN-LT has been proposed. The generator has the characteristics of compact structure, modularization, and repetitive operation. The output voltage pulse on a nominal $12 \Omega$ high-power solid resistor is $245 \mathrm{kV}$ with a pulse width of $180 \mathrm{~ns}$. The output power is calculated to be about $5.2 \mathrm{GW}$, which is corresponding to the power of four modules of the generator in [1]. The experimental results on this generator prove the feasibility to improve the output power of the module, which established a foundation to develop a pulse generator with a higher output power.

\section{Data Availability}

The data used to support the findings of this study can be made available upon request to the corresponding author.

\section{Conflicts of Interest}

The authors declare that they have no conflicts of interest.

\section{References}

[1] M. J. Li, Q. Kang, J. Tan, F. Q. Zhang, M. Luo, and F. Xiang, “A repetitive long-pulse power generator based on pulse forming network and linear transformer driver," Review of Scientific Instruments, vol. 87, Article ID 064704, 2016.

[2] A. A. Kim, B. M. Kovalchuk, V. A. Kokshenev et al., "Review of high-power pulsed systems at the institute of high current electronics," Matter and Radiation at Extremes, vol. 1, no. 4, pp. 201-206, 2016.

[3] S. D. Korovin, V. V. Rostov, S. D. Polevin et al., "Pulsed power-driven high-power microwave sources," in Proceedings of the IEEE, vol. 92, no. 7, pp. 1082-1095, 2004.

[4] A. Lindblom, M. Elfsberg, T. Hurtig, A. Larsson, and S. E. Nyholm, "First trials with a $45 \mathrm{GW}$ cable-based pulsedpower generator," Acta Physica Polonica A, vol. 115, no. 6, pp. 976-977, 2009.

[5] B. M. Novae, M. Istenic, J. Luo et al., "A $10 \mathrm{GW}$ pulsed power supply for HPM sources," in Proceedings of the 16th IEEE International Pulsed Power Conference, pp. 358-361, Albuquerque, NM, USA, June 2007.

[6] E. Schamiloglu, R. J. Barker, M. Gundersen, and A. A. Neuber, "Modern pulsed power: Charlie Martin and beyond," in Proceedings of the IEEE, vol. 92, no. 7, pp. 1014-1020, 2004.

[7] P. Wang, F. Xiang, J. Tan, M. Luo, Q. Kang, and G. P. Wang, "A repetitive electron beam driver based on 6-stage linear transformer driver cavities," Review of Scientific Instruments, vol. 91, Article ID 044705, 2020.

[8] B. Z. Zhang, F. Xiang, J. Tan, and A. B. Chang, "Simulation research on feed modes of linear transformer driver," High Power Laser and Particle Beams, vol. 22, pp. 2769-2772, 2010, in Chinese.

[9] H. Li, H.-J. Ryoo, J.-S. Kim, G.-H. Rim, Y.-B. Kim, and J. Deng, "Development of rectangle-pulse marx generator based on PFN," IEEE Transactions on Plasma Science, vol. 37, no. 1, pp. 190-194, 2009. 
[10] Q. Wu, Y. Cui, J. Gao, S. Li, and H. Yang, "A High-voltage pulse generator based on PFN and varistors," IEEE Transactions on Plasma Science, vol. 47, no. 1, pp. 512-517, 2019.

[11] H. Hertwig and Y. Li, Induction Calculation, National Defense Industry Press, Bejing, China, 1960, in Chinese. 